\title{
Steuerfreie Bonbons für geschätzte MFAs
}

Gute Mitarbeiter kann man durch besondere Zuwendungen besser an die Arztpraxis binden. Damit das Finanzamt nicht den größten Teil davon kassiert, sollte man die steuerrechtlichen

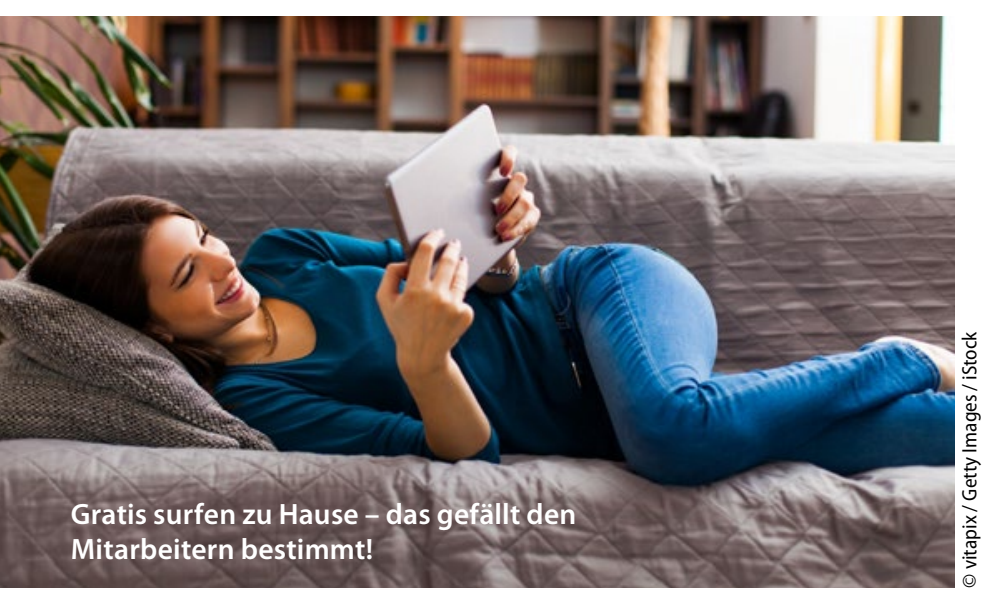

Freiheiten nutzen, die es hier (noch) gibt. In Betracht kommen z. B. ein Zuschuss zum Fitness-Center oder die kostenlose Nutzung von Arbeitsgeräten.

MMW-KOMMENTAR
Die Privatnutzung von
Arbeitsgeräten wie Lap-
tops, Smartphones oder
Tablets ist für Mitarbeiter
der Praxis steuerfrei -
selbst wenn sie nach
Hause mitgenommen
werden. Grundlage ist
eine Gesetzesänderung,
die am 8. Mai 2012 im
Bundesgesetzblatt I auf
Seite 1.030 veröffentlicht
wurde. Mit ihr wurde die
Steuerbefreiungsvor-

schrift nach § 3 Nr. 45 EStG deutlich ausgedehnt.

Steuerfrei sind nach dieser Norm die Vorteile der Angestellten aus der privaten Nutzung von betrieblichen Datenverarbeitungs- und Telekommunikationsgeräten. Dazu zählt auch deren Zubehör, also auch die zur privaten Nutzung überlassenen Programme und Apps, die der Arbeitgeber in seinem Betrieb einsetzt. Auch Grundgebühr und Verbindungsentgelte müssen nicht versteuert werden, ebenso wenig wie die Wartung und andere Dienstleistungen für das Gerät. Es ist auch unerheblich, in welchem Umfang eine private Mitbenutzung stattfindet.

Dies bedeutet, dass auch dann kein steuerpflichtiger Arbeitslohn entsteht, wenn die MFA die zur häuslichen Nutzung leihweise überlassenen Geräte oder Programme ausschließlich privat nutzt.

\section{EBM-Änderungen zum iFOBT beachten}

_ Vor Kurzem wurde mit dem iFOBT ein neuer Test zur Suche nach verstecktem Blut im Stuhl eingeführt (siehe MMW 5/2017, S. 25). In diesem Zusammenhang wurde nun im EBM die Kostenpauschale nach Nr. 40150 für die Ausgabe von guajakbasierten Stuhlbriefchen, die nicht zurückgegeben wurden, verändert. Entfernt wurde der Verweis auf die Nr. 01734 entfernt, da diese zum 1. April weggefallen ist.

\section{MMW-KOMMENTAR}

Die Nr. 40150 selbst kann noch bis zum 30. September 2017 berechnet werden, wenn der Leistungsinhalt der Nr. 32040, die Untersu- chung auf Blut im Stuhl in drei Proben, nicht erfüllt werden konnte, weil die Stuhlbriefchen vom Patienten nicht zurückgebracht wurden. Ab dem 1. Oktober 2017 entfällt dann auch die Abrechnung dieser Positionen, da nur noch der iFOBT Kassenleistung ist und dieser nur von berechtigten (Labor-)Ärzten abgerechnet werden kann.

\section{Neue Qualitätsregeln für Sonografie}

_ Seit 1. April 2017 gelten neue Regeln für die Hygienequalität bei Endosonografiesonden. Nach $\$ 9$ Abs. 4 der Ultraschall-Vereinbarung (US-V) müssen die Hersteller nun in der Gebrauchsanweisung ein wirksames und materialverträgliches Desinfektionsverfahren angeben, das auch durch ein Gutachten belegt sein muss. Das Bundesinstitut für Arzneimittel und Medizinprodukte (BfArM) hatte eine solche Regel bereits 2005 empfohlen.
Die Hersteller können allerdings bisher kein solches Gutachten vorlegen. KBV und GKV-Spitzenverband haben deshalb nun eine Übergangsregelung bis zum 31. September verlängert. Das BfArM wurde einstweilen gebeten, die formalen und inhaltlichen Voraussetzungen an das Gutachten zu erläutern.

\section{MMW-KOMMENTAR}

Im gleichen Zuge haben KBV und Kassen eine Unsicherheit hinsichtlich der Ultraschall- kurse ausgeräumt, mit denen man die fachliche Befähigung für Sonografie erlangt. Hier ist vorgeschrieben, dass der Kursleiter die Teilnahme durch ein "Zertifikat" bestätigen muss - verschiedene Kursanbieter stellen stattdessen aber "Teilnahmebescheinigungen" aus. Durch eine Anpassung des § 6 US-V wurde nun klargestellt, dass es sich hier um eine legitime alternative Form der Bestätigung handelt.

Dr. Gerd W. Zimmermann, Hofheim 